\title{
AdAPTIVE CONTROL SySTEM SYNTHESIS METHODS FOR COMPleX MANUFACTURING OBJECTS
}

\author{
Nikita S. Kudriashov, Sergey A. Markov and Vyacheslav V. Potekhin \\ Peter the Great St. Petersburg Polytechnic University
}
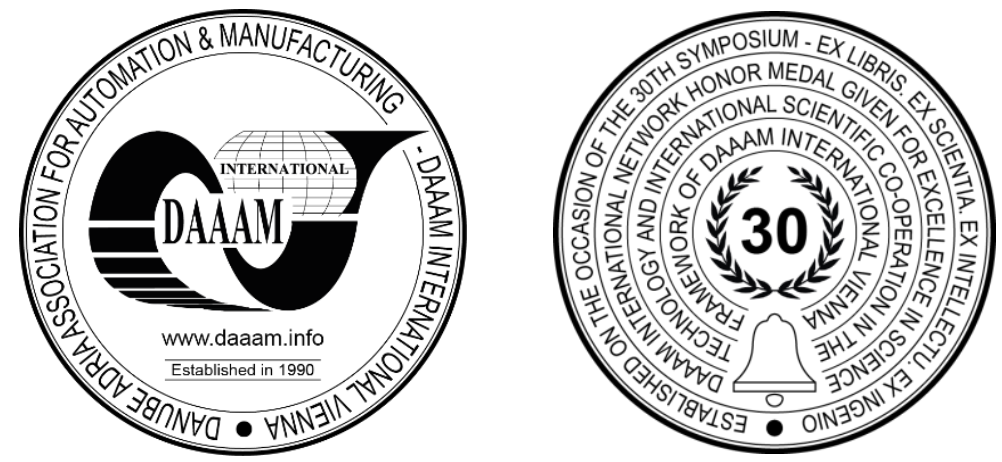

This Publication has to be referred as: Kudriashov, N[ikita]; Markov, S[ergey] \& Potekhin, V[yacheslav] (2019). Adaptive Control System Synthesis Methods for Complex Manufacturing Objects, Proceedings of the 30th DAAAM International Symposium, pp.0493-0499, B. Katalinic (Ed.), Published by DAAAM International, ISBN 978-3-90273422-8, ISSN 1726-9679, Vienna, Austria

DOI: $10.2507 / 30$ th.daaam.proceedings.066

\begin{abstract}
In this paper, the authors present the analysis of development of the adaptive control system for complex manufacturing objects. As a result, paper shows a relevance of digital twin's concept to provide such control, and its main features. This work starts from the determination of the borders between simple and complex manufacturing objects, in which usual control approaches are not relevant. In that way the paper shows the main features of the control objects, for which adaptive control systems are needed. Using such analysis as a basement, the main features of such adaptive control systems was discovered. Although, the authors created and describe the main scenarios, which are needed to provide the suitable control of such complex systems. Summarizing previous results, the authors present requirements for digital twin - as an object, which provides all the needed functionality. In particular, authors focus on infrastructure needed to make the digital twin work. Then the digital twin's complete functional scheme is described. Actuality of this paper is to present an approach to adaptive control systems development, based on manufacturing objects needs all around the modern world.
\end{abstract}

Keywords: Industry 4.0; digital twin; adaptive control; model predictive control; Machine learning; Cyber-Physical Systems.

\section{Introduction}

According to global digitization trends and Industry 4.0 paradigm, modern world dictates us more and more new requirements for control systems, such as:

- Control systems have to be widely flexible and adapt to any technological process changes. The spreading of IoT solutions makes the most contribution in this case.

- Provide control, based not only on real but prognosis data for technological parameters. Basically connected with the growth of the interest to high level planning systems.

- Control system has to provide the optimal regime of work (energy consumption, equipment durability, profit etc.)

These are the main features of the new control system and the main strategies of the companies all around the globe. Such companies, mostly, solve this problems by building adaptive control systems, based on, so called, digital twins of 
manufacturing objects - the basement for wide range of the control scenarios. In spite of it, the problem is that there is still no conventional approach to such development ${ }^{[1]}$.

There are many papers, describing main concepts of adaptive control systems and digital twin development process. Most of them describe the solution of some concrete engineering problem, for specific field of some typical technological objects. ${ }^{[2,3]}$. Problem is that there is a lack of works, concentrated on fundamentals of digital twins development. ${ }^{[4]}$ That is the reason, why most of the engineers start from the basics, all the time developing the digital twin.

In this paper we present approach to development of adaptive control system and digital twins for complex manufacturing objects. This generalized approach can be applied as the basement for more narrow fields. It is forwarded to connect all the needs of nowadays industry and summarize previous scientific results. This approach has to resolve such questions as.

- Where is the border between simple and complex manufacturing objects, in which usual control approaches are not relevant.

- What is the main features and functionality of such adaptive control systems and the main features of digital twins.

- What are the requirements for digital twin - as an object, which provides all of the needed functionality for adaptive control system.

- What algorithms have to be applied to realize all of the needed control scenarios.

On the Fig. 1, two approaches to control system architecture are described. On the left - the simple control system, on the right -the generalized adaptive control system for complex manufacturing object.
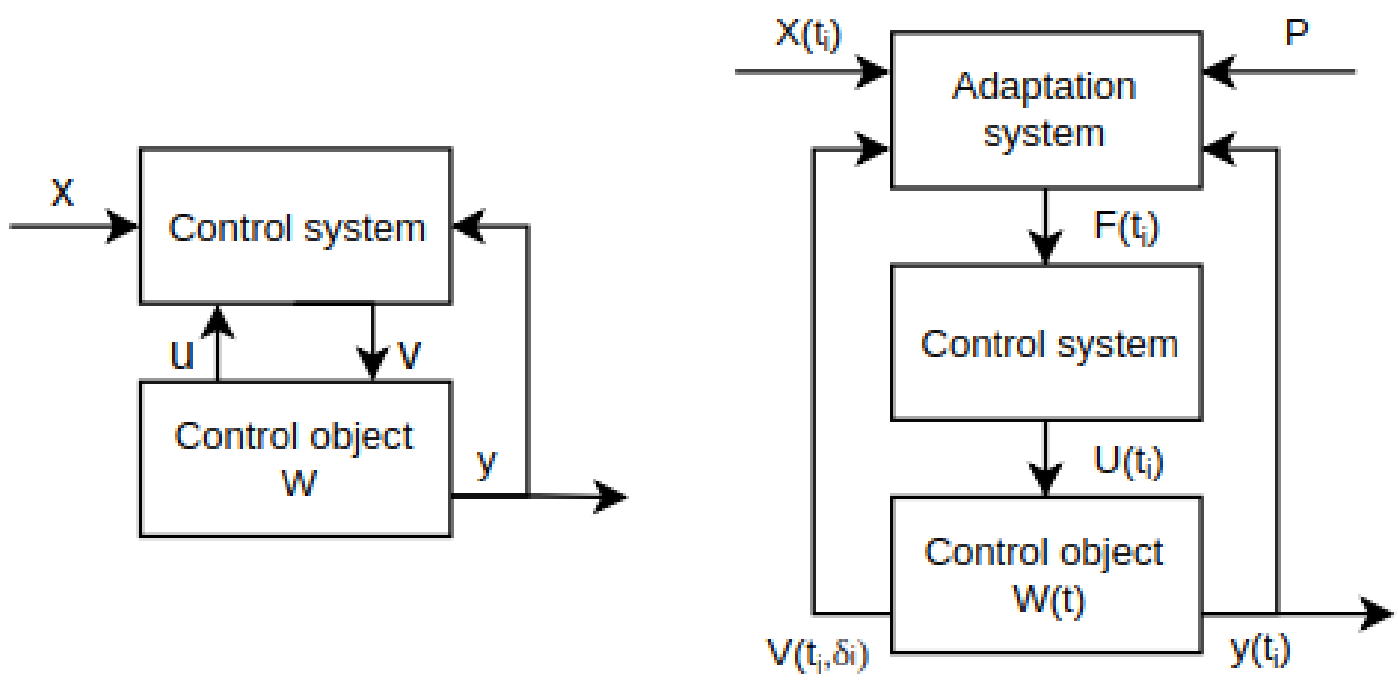

Fig. 1. Control system architectures, where $X\left(t_{i}\right)$ - input control data in $t_{i}$ moment of time, $\delta_{i}-$ uncertainty value; $U-$ set points from control system to control object; $\mathrm{V}$ - signals from object, such as sensor data; $\mathrm{Y}\left(\mathrm{t}_{\mathrm{i}}\right)$ - controlled parameter; $\mathrm{W}(\mathrm{t})$ - control object characteristic, $\mathrm{t}$ means it changes during the time; $\mathrm{F}\left(\mathrm{t}_{\mathrm{i}}\right)$ - adjustment data, provided to change the control parameters

Although, $\mathrm{F}\left(\mathrm{t}_{\mathrm{i}}\right)$ contain $\mathrm{X}\left(\mathrm{t}_{\mathrm{i}}\right), \mathrm{Y}\left(\mathrm{t}_{\mathrm{i}}\right)$, V, this values is needed to provide control and can be connected from the field level strait into the control system, or can be transferred through the Adaptive system, $\mathrm{P}$ - data from other object, systems (plan or set-points). $\mathrm{F}\left(\mathrm{t}_{\mathrm{i}}\right)$ depends on data provided by digital twin and real data from the object. Format of such data depends on control system algorithms format. According to this data, adaptive control system have to provide appropriate control scenarios. For this paper we assume that the structure of the controller is constant, so $\mathrm{F}\left(\mathrm{t}_{\mathrm{i}}\right)$ contains only its parameters and coefficients ${ }^{[5]}$.

\section{Control system requirements}

The term Complexity in this paper is a criterion which generalize such features of manufacturing object, that make it more difficult to control. In this case we use this criterion to determine such objects, for which standard control algorithms will not work properly or can't be applied at all. Although, we apply this criteria as the problems to be solved, while creating complex manufacturing object adaptive control system ${ }^{[6]}$.

The idea of development of an adaptive control system for complex object is to determine the complexity at the first step. After that we transfer complexity values into control system requirements. In this paper we describe the objects, 
which contains the high grade of all of the complexity features. In this way, the complexity of manufacturing process can be rated due to next features, shown below.

Multi-variable or multi-connected control object. Such systems in which control is carried out to several connected variables. Moreover, all input variables have a significant effect on the state of the entire object, and their interaction is manifested in all output parameters of the object.

Let us assume that in usual strait objects, dependence between input $\mathrm{X}_{\mathrm{n}}$ and output $\mathrm{Y}_{\mathrm{k}}$ can be described by $\mathrm{W}_{\mathrm{nk}}$ transmission function. At the same time $\mathrm{Y}$ in musty-connected control object description is more complex. On the Fig. 2. such connection is shown.

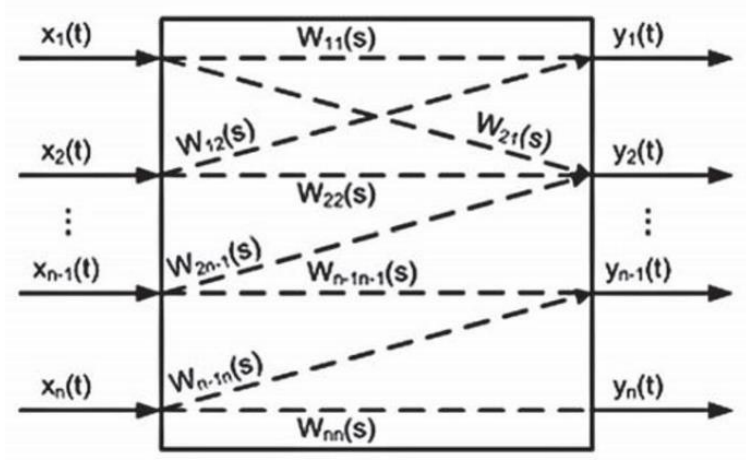

Fig. 2. Multi-variable control object

Here we can see that $\mathrm{Y}_{2}$, for example depends on $\mathrm{X}_{1}, \mathrm{X}_{2}, \mathrm{X}_{\mathrm{n}-1}$ inputs and $\mathrm{W}_{12}, \mathrm{~W}_{22}, \mathrm{~W}_{\mathrm{n}-\mathrm{n}-1}$ transmission functions. Because of described features, arises the complexity of management of such systems. It appears an additional control tasks, such as:

- Negative relative value influence elimination.

- Dynamic diagnostics of an object characteristic or system state.

- Control parameters optimization, according to set points

Continuous manufacturing process is a flow production method working without interruption. It applies more rigid requirements to process development and description. For manufacturing objects, based on such process it is very important not to fall into alarm situation and stop. Because of such sensitivity, control systems for such objects has to meet such requirements as:

- Applying of in-line, on-line or at-line measuring techniques

- High ratio of control sequences development

- Prediction of alarm situations and breakdown prevention.

High level of integration of the process with other systems. Such features make object more complex first of all because of arising number of constraints, provided by adjacent objects. It makes object more Multi-variable and increases the computation loads. We can highlight such provided requirements as:

- Control parameters optimization, according to objects states.

- High time ratio requirements due to synchronization constraints.

- Analysis of cause and effect of the plan and control

Uncertainty of an object. For example, distillation column in oil manufacture - one of such difficult manufacturing object. Even if it will be described completely, there is no guaranty that sensors and equipment possible to be provided to satisfy the description. In such cases control object can be described as the dynamically variable. So the main control system requirements for this parameter are:

- Dynamic identification of the object in its current state

- Multi-model approach to description (Description of an object with several heterogeneous models)

Incompleteness of the sensor data. As an example, we can consider the same distillation column. Some of parameters, needed to provide the control (such as composition of petrol) cannot be evaluated with required frequency because of specific laboratory analysis necessity. In this case adaptive control system has to provide such feature as: parameters reconstruction and parameters prediction

High degree of object loading. Modern world dictates higher speeds, quality and volumes of manufacturing, and this needs growing faster every year. Such speeds do not allow us to establish new manufacturing workshops every time 
manufacture need to speed-up. Industry 4.0 concept provides much approaches to optimization of manufacture to solve such problem, we consider main of them:

- Equipment loads distribution and optimization.

- Planning, scheduling and prediction of processes values.

Every complexity feature above contains some basic requirements to control system, which should handle with such objects. Now we summarize them into list of general features. In Fig. 3. such process is illustrated in form of graph, which links the features into requirements, which have to be realized in adaptive control system.

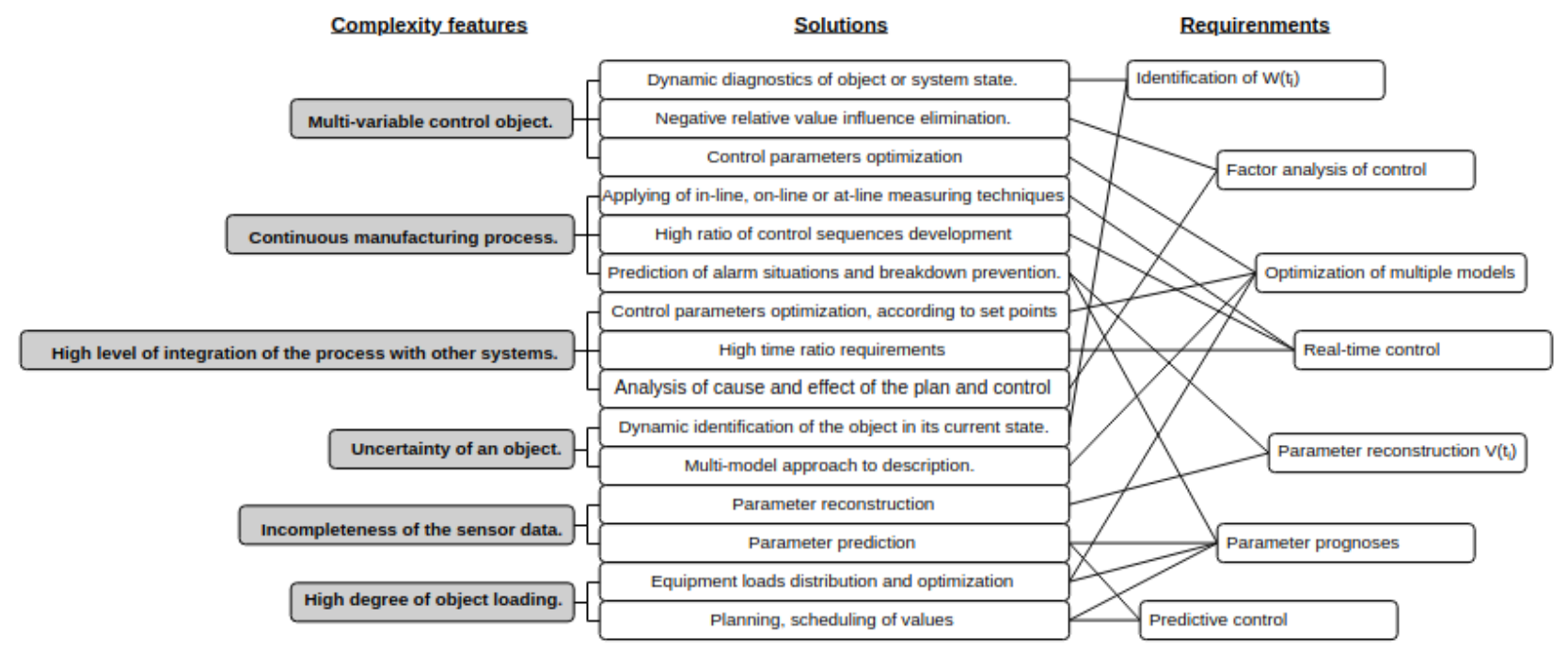

Fig. 3. Adaptive control system requirements

In this case, adaptive control system requirements are:

- Control system have to provide the control, based on the identified object $\mathrm{W}(\mathrm{t})$.

- Control has to be based on constraints from factor analysis of the control system.

- Multi-model approach is needed to find the best W(t) for control.

- Control system has to work in a real time mode.

- Parameters V has to be reconstructed and interpolated

- System has to provide prediction of parameters of the system.

- Predictive control algorithms has to be provided ${ }^{[7]}$

\section{Adaptation system structure}

Previously in this paper, we have developed the main features of the complex manufacturing object. At the same time we stated, that adaptive control system, should provide appropriate control, based on model data from so-called Digital Twin of an object.

On the Fig. 1. we have shown an architecture of adaptive control system, in which the adjustment parameters F(ti) are provided. Now, when we've described control system requirements, we can develop an adaptation system architecture and control scenarios. It is shown on the Fig. 4. One of the advantages of the structure, that it can be reduced due to specifics of an object (in this paper can be evaluated through the complexity).

An adjustment module idea is taken from the classic adaptive control system theory. Specification - parameters which describes the way module will work. The set of modules and scenarios can be set via these parameters. Adjustment model connects to Digital Twin to take the whole information about the control system, to provide the adjustment data.

Identification module - solves the homonymous problem in requirements. It takes raw $\mathrm{V}$ data from sensors and $\mathrm{y}$ feedback values and predict the $\mathrm{W}\left(\mathrm{t}_{\mathrm{i}}\right)$. Digital Twin provides the model data to make such identification more precise. This can be reached using such models as:

- The physical model of an object - describing the offered W matrix structure.

- Mathematical model of an object - describing the physical constrains for the object. 


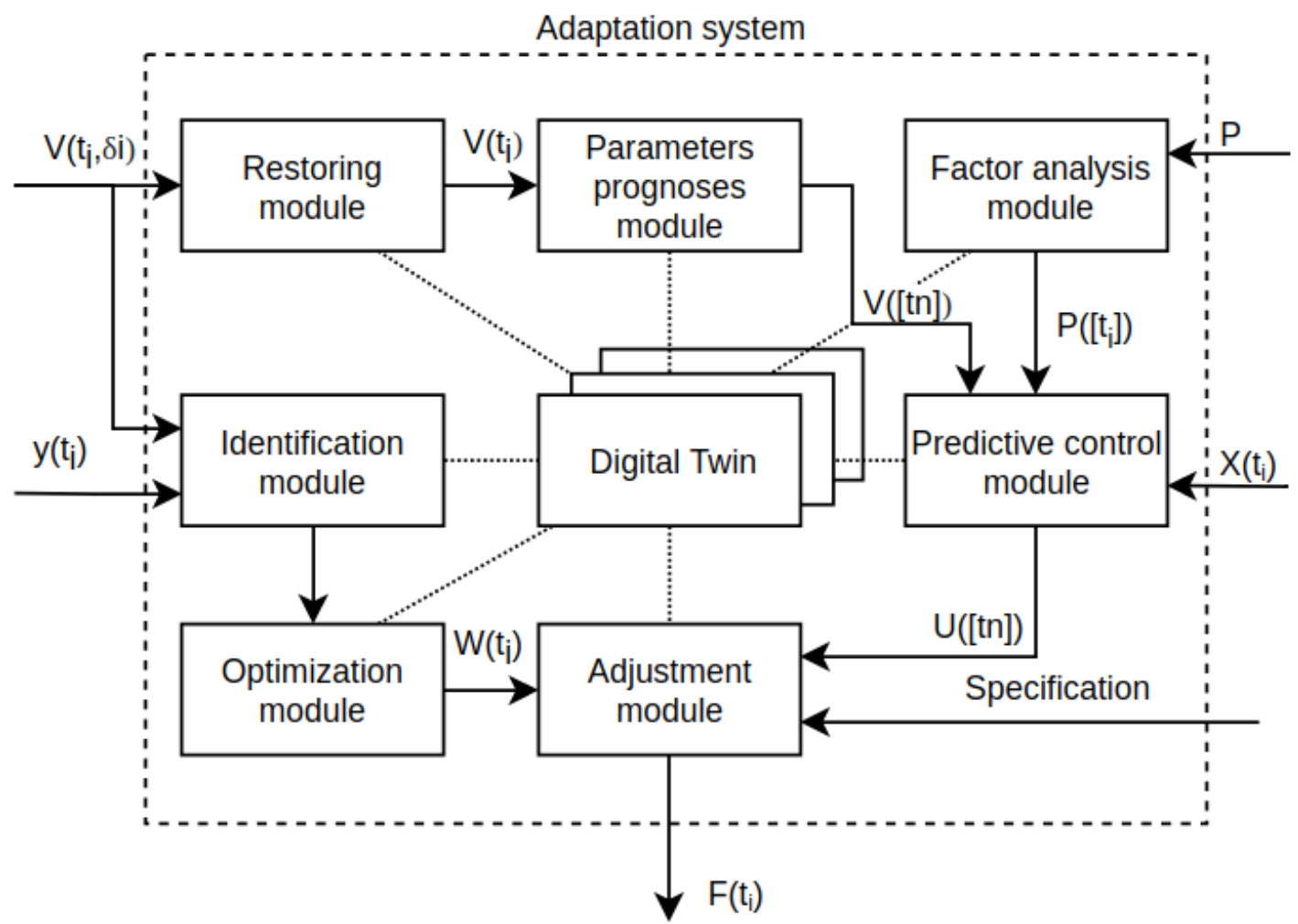

Fig. 4. Adaptation system

The identification result $\mathrm{W}_{1}\left(\mathrm{t}_{\mathrm{i}}\right)$ goes into Optimization module. At the same time Digital Twin can handle the number of heterogeneous models. They provide $\mathrm{W}_{\mathrm{n}}\left(\mathrm{t}_{\mathrm{i}}\right)$ values to calculate the optimal $\mathrm{W}$ to fit into $\mathrm{Y}$ and $\mathrm{V}$ and Adaptation system specification. So the best one to be chosen as $\mathrm{W}\left(\mathrm{t}_{\mathrm{i}}\right)$ for adjustment. As a simplest example:

- Saved $\mathrm{W}\left(\mathrm{t}_{\mathrm{i}-1}\right)$ from previous identification iteration, can be compared to $\mathrm{W}_{1}\left(\mathrm{t}_{\mathrm{i}}\right)$.

- Statistical models - ML-based models, describing W in many variable ways (decision trees, coefficient matrices, Li-transforms etc.)

- $\quad$ The physical model of an object provide $\mathrm{W}$, based on physical modeling of an object.

To handle the prediction and planning requirement, adaptation system has to take into attention the predicted $U\left(\left[t_{n}\right]\right)$ or predicted $\mathrm{V}\left(\left[\mathrm{t}_{\mathrm{n}}\right]\right)$. This is handled with restoring, parameters prognoses and predictive control module. Restoring is the process of deliverance from the $\delta$ uncertainty of sensors data. It can be, for example Interpolation or extrapolation of data supported with: Statistical ML models, Physical models, Mathematical models. The same type of models are used to predict the future values $\mathrm{V}\left(\left[\mathrm{t}_{\mathrm{n}}\right]\right)$ for the specified planning horizon $\mathrm{n}$, based on restored $\mathrm{V}$ values. Although, CAD models can be applied to predict the margins of safety for the equipment, what will be needed in Predictive control module. ${ }^{[8]}$

Factor analysis module is needed to provide constraints, limitations and parameters of control. This module process $\mathrm{P}$ data, which is the high level data, containing:

- Key performance indicators (KPI)

- $\quad$ Set-points from planning systems

- Energy efficiency for manufacturing parameters

- $\quad$ Resources limitations

Using such information, factor analysis module provide the optimal parameters for adjustment for predictive control $\mathrm{P}\left(\left[\mathrm{t}_{\mathrm{n}}\right]\right)$. For example, same set point in the future can be reached withing different control scenarios, but most of them would not fit into P, that is why it is important to evaluate such P. Module uses control model and CAD model to find such values.

Using $\mathrm{V}\left(\left[\mathrm{t}_{\mathrm{n}}\right]\right)$ as a prognosis of object parameters and $\mathrm{P}\left(\left[\mathrm{t}_{\mathrm{n}}\right]\right)$ as a set of constraints, we can evaluate the prognosis of set points from control system to control object $U\left(\left[t_{n}\right]\right)$. Control module uses such approaches as MPC, LQR etc. All of such scenarios need the control model to be provided by digital twin. $\mathrm{X}\left(\mathrm{t}_{\mathrm{i}}\right)$ is the set-point for control system and is needed to provide the U. Otherwise, when the system is integrated with high-level planning systems, such value can be found in $\mathrm{P} \mathrm{data}^{[9]}$.

All of the requirements was performed except one - control system has to work in a real time mode. Let us assume that time between $F\left(t_{i}\right)$ and $U\left(t_{i}\right)$ is $t_{c}$. In the simple control systems (where instead of $F$ there are $x, y$ and $v$ ) this time has 
to be less than object measurement and set point generation frequency. In our case we have to achieve the same value but for time $t_{a}+t_{c}$. Time $t_{a}$ is the time between $F\left(t_{i}\right)$ generation and $v, y$, $x$ values emergence ${ }^{[10]}$.

$\mathrm{V}$ values going into restoring, prognoses, factor analysis, predictive control modules can be evaluated in parallel with other processes. Therefore, we have two threads, which should fit into $t_{a}$ time margins: identification-optimisation and restoring-control segments. It can be implemented through the cloud computing technologies. Especially, interaction with digital twin (as a main process of any module of the adaptation system) time coasts need to be minimised.

\section{Digital Twin structure and requirements}

We've already described the main features of an adaptive control system for complex manufacturing object. We've presented the architecture of the adaptation system, every element of which is linked to so-called digital twin. Such digital twin has to provide prepossessed multi-model, and do it in minimum time period as possible. Therefore, in this part we suggest the structure of the digital twin and describe some basic requirements, as a result of this paper.

During the $3^{\text {rd }}$ part of the paper, we've noted models needed for every module of the adaptation system:

- Mathematical models - description based on the mathematical comprehension of the system.

- Physical models - description using physical processes inside of an object (Hysys, Petrosim etc.).

- Statistical models - models based on statistical data from the object (Machine learning algorithms).

- $\mathrm{CAD} / \mathrm{CAE}$ models - technical description of an object.

- Control models - description of the control algorithms for the object

Creation of set of models is not only one think we need to pay attention to. More important is to provide the access to model data. On Fig. 5. an example of the digital twin architecture is described ${ }^{[11]}$.

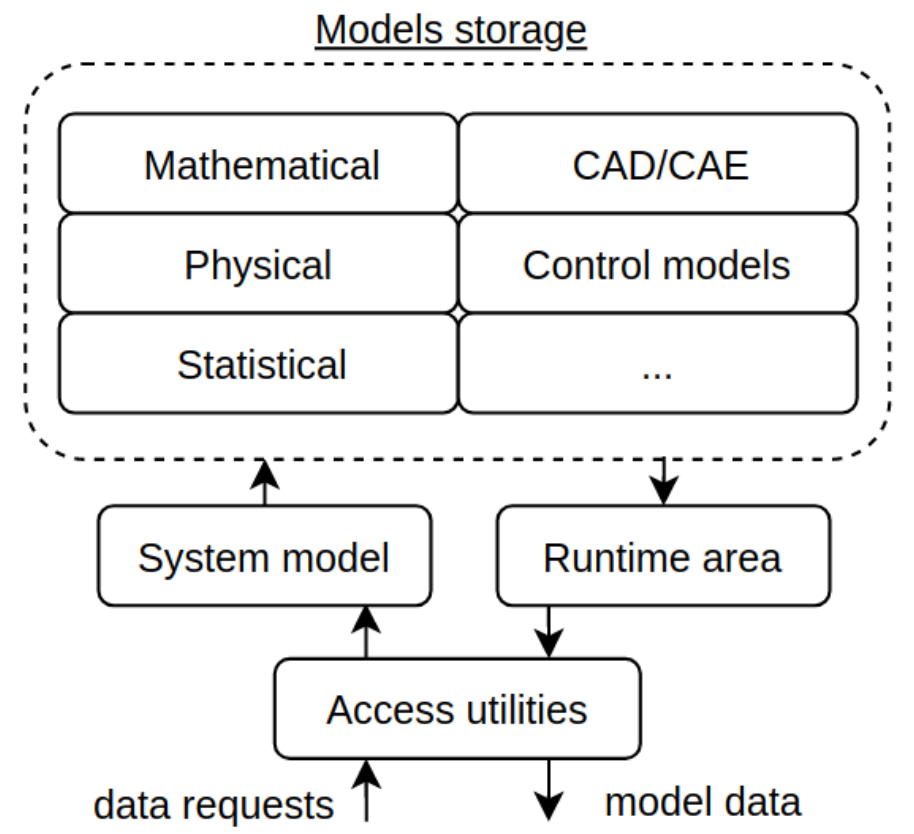

Fig. 5. Digital twin structure

To provide a rapid access to model data, at first we need to somehow link all the models together. For example, if we need to run the restoring module (statistical and physical models are needed) for some value of an object we need to route the request into those models. That is where system models come up.

Such system models have to describe an object from many sights, such as functionality, structure, behavior etc. Such requirements are identical to UML notation goals. The Unified Modeling Language (UML) is a general-purpose, developmental, modeling language in the field of software engineering that is intended to provide a standard way to visualize the design of a system. In this way such language not only provides the access to needed model, but helps to develop them.

Such notation cannot work by itself. It just provides fast linking, updating and execution of models. To realize evaluations, digital twin has to provide the area for models launches - runtime area. Structure of such area depends from model set, but it always has to provide possibility to run heterogeneous models. 
When model data is requested, according to adaptation system requirements, access utilities transfer such requests into system model, which links them into model storage. Required models from the storage transfers into runtime area which is based on specifications of the models (such as modeling systems runtimes, computation optimisation algorithms etc.). Runtime area provides data back into access utility, and it transfers back into adaptation system.

Withing such architecture, we provide the realization of all digital twin requirements.

\section{Results}

In this paper authors have analyzed the main features of the complex manufacturing objects. Such features were used to provide the functionality and requirements for adaptive control system. To realize required functionality, authors developed an architecture of an adaptation system for adaptive control. Digital twin structure was suggested as an object, to provide model data about the control object, to adjust the parameters of the control system. Result of this paper is the generalized structure of adaptive control system for complex technological objects and the set of requirements to it.

All of the research questions have been answered.

- Border between simple and complex manufacturing objects has been described as a set of values to be evaluated.

- Main features and functionality of adaptive control systems and the main features of digital twins have been performed in this paper.

- Digital twin requirements was developed in order to provide all of the needed functionality for adaptive control system.

- Set of algorithms has been offered to realize all of the needed control scenarios.

Further works in this area are: find the best hardware and software solution for such system and test it on a real manufacturing objects.

\section{Acknowledgment}

The paper is published in the framework of the project Erasmus+ 573545-EPP-1-2016- 1-DE-EPPKA2-CBHE-JP and describes the part of the project conducted by Peter the Great St. Petersburg Polytechnic University.

\section{References}

[1] B. Katalinic, I. Kukushkin, V. Pryanichnikov, D. Haskovic, Cloud Communication Concept for Bionic Assembly System, Procedia Engineering, Volume 69, 2014, Pages 1562-1568, ISSN 1877-7058.

[2] M. A. Al Faruque and F. Ahourai, "A model-based design of cyber-physical energy systems," in Design Automation Conference (ASP-DAC), 2014 19th Asia and South Pacific, pp. 97-104, IEEE, 2014.

[3] Negri, E.; Fumagalli, L. \& Macchi, M. A review of the roles of Digital Twin in CPS-based production systems, Procedia Manufacturing, Volume 11, 2017, Pages 939-948.

[4] Ponomarev, K.; Kudryashov, N. \& Popelnukha, N. (2017). Main Principals and Issues of Digital Twin Development for Complex Technological Processes, Proceedings of the 28th DAAAM International Symposium, pp.0523-0528, B. Katalinic (Ed.), Published by DAAAM International, ISBN 978- 3-902734-11-2, ISSN 1726-9679, Vienna, Austria.

[5] Scheifele, S., Friedrich, J., Lechler, A. and Verl, A. (2014) 'Flexible, Self-configuring Control System for a Modular Production System', Procedia Technology. Elsevier B.V., 15, pp. 398-405. doi: 10.1016/j.protcy.2014.09.094.

[6] Kudriashov N., Protasov I., Markov S., Potekhin V., Yadgarova Y., Taratukhin V. (2016). Implementation of Cloud Services for Advance Management of Steel Transport for Continuous Casting Production, Proceedings of the 27th DAAAM International Symposium, pp.0457-0462, B. Katalinic (Ed.), Published by DAAAM International, ISBN 978-3-902734-08-2, ISSN 1726-9679, Vienna, Austria

[7] Nikolic, B.; Ignjatic, J.; Suzic, N.; Stevanov, B. \& Rikalovic, A. (2017). Predictive Manufacturing Systems in Industry 4.0: Trends, Benefits and Challenges, Proceedings of the 28th DAAAM International Symposium, pp.07960802, B. Katalinic (Ed.), Published by DAAAM International, ISBN 978-3-902734-11-2, ISSN 1726-9679, Vienna, Austria DOI: 10.2507/28th.daaam.proceedings.112.

[8] Dutta, S.; Bandopadhyay, S.; Ganguli, R. \& Misra, D. Machine Learning Algorithms and Their Application to Ore Reserve Estimation of Sparse and Imprecise Data, J. Intelligent Learning Systems \& Applications, 2010, Volume 2, Pages 86-96.

[9] Negri, E.; Fumagalli, L. \& Macchi, M. A review of the roles of Digital Twin in CPS-based production systems, Procedia Manufacturing, Volume 11, 2017, Pages 939-948.

[10] J. Lee, E. Lapira, B. Bagheri, H. an Kao, Recent advances and trends in predictive manufacturing systems in big data environment, Manuf. Lett. 1 (2013) 38-41. doi:10.1016/j.mfglet.2013.09.005.

[11] A. Canedo, Industrial IoT Lifecycle via Digital Twins, in: Proc. Elev. IEEE/ACM/IFIP Int. Conf. Hardware/Software Codesign Syst. Synth., 2016: p. 29. 